\section{Dosimetry of paediatric radiological and scintigraphic studies of the foregut}

\section{S Wynchank}

\section{D Mann}

Institute of Child Health and University of Cape Town

\section{Introduction}

The two principal imaging modalities for study of paediatric gastrointestinal tract (GIT) function are scintigraphy and radiology. They differ in many respects, but their clearest common feature is that both use ionising radiation, hence both result in an absorbed radiation dose per unit mass of tissue (D). D is deleterious if too large. There is no single value denoting acceptable levels of $\mathrm{D}$, as tissue sensitivity varies. So all imaging should be undertaken after consideration of its benefits and risks, although the former are difficult to quantify. ${ }^{1}$ Emphasis here is on quantitation of $D$, for it is important to know this value at different sites.

There are significant differences between paediatric and adult patients when considering dosimetry. The former live longer, so are subject to greater risk of any late manifestations of radiation. Paediatric organs have age-dependent mass ratios, which can differ from those of adults, most especially for brain, testes and adrenals. An additional important determinant is the increased sensitivity to ionising radiation of some paediatric tissues, especially tissues associated with reproduction and rapid growth. Uptake and retention of radiopharmaceuticals in different organs may exhibit different physiology from that of adults (most notably in the growth areas of long bones). Hence dosimetric considerations in paediatrics are not those of miniature adults.

\section{GIT scintigraphy}

Scintigraphic images are obtained after detection of gamma rays recorded by a gamma camera next to the patient. These gammas are emitted by radioactive nuclei located within the patient. The radionuclei are attached to a molecule (radiopharmaceutical), chosen so that it performs a desired function. Administration of the tracer in GIT studies is usually by deglutition. The selected radiopharmaceutical for a specific study is chosen to concentrate in an organ, tissue or system. Then images of its distribution are recorded. Often the radiopharmaceutical takes part in a physiological activity, either intra- or extracellular, so scintigraphy indicates function. In GIT studies of a swallowed tracer, the function observed is bolus motility. Thus it is clear that the $\mathrm{D}$ value after scintigraphy depends only on the size and physical characteristics of the administered radiation dose and its subsequent course (i.e. its spatial distribution within the body and residence times in different regions). The time spent recording scintigraphic images bears no relation to $\mathrm{D}$, but is limited solely by availability of personnel and equipment and the value of the resulting data. Observations can therefore be made for much longer than in radiology.

Scintigraphy's spatial resolution is almost an order of magnitude below that of radiology, although this difference is being steadily reduced with technical improvements. D values are usually much smaller for scintigraphy than radiology. In contrast to radiology, scintigraphic images provide information on the presence of each individual contributing molecule in the radiopharmaceutical, so the images are quantifiable. In gastrooesophageal reflux (GOR) studies, activity in each reflux, and by inference the volume of refluxate, can easily be obtained and many studies have shown that scintigraphy has greater sensitivity in the detection of this. ${ }^{23}$ Overall, scintigraphy is a quantitative, functional modality, with some morphological characteristics. It is therefore the complete opposite of radiology, which is mainly morphological and much more qualitative. Hence the two modalities are complemen- 
tary in terms of the information they can provide.

In the scintigraphic study derived for use at this institution (Red Cross War Memorial Children's Hospital), the following information is obtained. The movement of a swallowed bolus comprising a few millilitres of liquid down the oesophagus is recorded. Possible dysmotility, which can be observed and quantitated, includes abnormally slow passage, holdup, retrograde motion, breakup of the bolus, and nasopharyngeal or laryngeal entry. Thereafter the balance of the feed without tracer is swallowed. This 'rinses' any particles of tracer which adhere to normal epithelium of an intact oesophagus. If the oesophageal epithelium is abnormal, such adherent tracer will remain. Most frequently this denotes oesophagitis and its extent and severity can then be deduced. Next, a search for GOR is undertaken, typically for 30 minutes. The height, duration and volume of each reflux are measured. Gastric contents at 30 and 120 minutes after swallowing are determined from the proportions of swallowed material in the stomach and in the distal GIT at these times. This presumes a thorough mixing of the tracer-containing material with all other stomach contents. Finally, at the time of the 120minute determination, a search for pulmonary aspiration is undertaken. As little as $0.2 \mathrm{ml}$ of swallowed liquid can be detected. ${ }^{4}$ If there is very strong suspicion of pulmonary inhalation of swallowed liquid, such a search can be repeated as often as required (without increasing D), for about 24 hours. This limit results from the physical half-life of Tc-99m of 6.04 hours. Gastric emptying may also impose a shorter limit.
It is therefore clear that the scintigraphic study is able to provide a great deal of information. It requires no unusual equipment or techniques and can be performed in any typical nuclear medicine department that possesses a computer-controlled gamma camera. The large amount of varied information available from this study is of considerable use, since many abnormalities detected (in particular, different forms of oesophageal dysmotility) may have the same symptoms as GOR. So in our experience inappropriate therapeutic decisions are frequently avoided by an accurate diagnosis provided by the scintigraphic study.

\section{Scintigraphic dosimetry}

In nuclear medicine procedures D depends on ionising radiation in a given (source) tissue or organ. Administered activity is measured in mega Becquerels ( $1 \mathrm{~Bq}=1$ disintegration/s). Emitted ionising radiation can have an effect in a (target) tissue or organ, other than its source. The absorbed dose rate is the amount of energy absorbed in the target region, per unit time, per unit mass, due to the ionising radiation. $\mathrm{D}$ is measured in Gray (1 Gy = 1 joule per kilogram) and depends directly on the rate of release of energy from the source region and the target region's absorption characteristics. (For example, a sufficiently large target region will absorb all radiation from the source that falls on it.) There are two conflicting factors in deciding how much radionuclide to administer. Advantages resulting from greater administered doses include better resolution of the resulting images and/or shorter duration of the study (important for some paediatric patients). Simultaneously there is an important disadvantage, for $\mathrm{D}$ increases in all tissues and organs. Careful dosimetric considerations allow an optimal balance between these factors when choosing the swallowed dose. Diagnostic nuclear medicine doses of radionuclides are generally low and the resulting diagnostic benefits typically exceed any possible risk. However, this may not be the situation with therapeutic doses, ${ }^{5}$ which is beyond the scope of this paper.

There is a commonly used and well proven method for calculating internal dose in nuclear medicine diagnostic techniques, which is that of the Medical Internal Radiation Dose (MIRD) Committee of the USA Society of Nuclear Medicine. ${ }^{6}$ The information given here is based on MIRD methods. Allowance is made for subjects of different ages and both sexes. $\quad$ Although other dosimetric procedures exist, they give results that rarely differ significantly from those of MIRD techniques. The MIRD method assumes that in a source region there is activity $\mathrm{A}$, whose magnitude depends on both physical and biological factors. This activity A, emitted isotropically, causes an absorbed dose $\mathrm{D}$ in the target region. The relation between $A$ and $D$ is given by $\mathrm{D}=\mathrm{A} . \mathrm{S}$. $\mathrm{S}$, the 'S factor', has been calculated for many pairs of source organs and targets, under a large variety of conditions. For paediatric dosimetry, $\mathrm{S}$ factors are calculated for average, normal children at various ages (usually $0,1,5,10$ and 15 years). The principles of these calculations are straightforward, but the resulting computation is often very complex, requiring multiple integrals, ${ }^{8}$ but there are computer programmes readily 
available which perform such dosimetric calculations.' In paediatric dosimetry age-dependent $\mathrm{S}$ factors are used. $^{10}$ If there is a non-uniform activity distribution in a source organ, then the resulting target organ doses can be inaccurate." However, this is rarely important in diagnostic nuclear medicine.

Different types of radiation may cause different biological effects and dosimetry calculations allow for this by using a quality factor $\mathrm{Q}$ (which is equal to 1 for $\mathrm{X}$ - and gamma-rays), so that the effective dose $(H)$ is defined by $\mathrm{H}=\mathrm{D} . \mathrm{Q}$. $\mathrm{H}$ is measured is Sievert (Sv).

The great majority of dosimetry studies have involved adults, so a common dosimetric practice for deriving doses administered to paediatric patients is to use a fraction of the adult dose. This is only satisfactory when the fraction is linked to clearly stated adult doses, for adult doses vary extensively. ${ }^{12-14}$ Administered paediatric doses based on age are inappropriate, but there is validity in using doses based on height, weight or surface area. ${ }^{15}$ However, recent work has calculated paediatric administered doses explicitly ${ }^{10.15}$ using the MIRD procedure with appropriate phantoms and this will be quoted in the present work.

In order to understand the dosimetry of swallowed radionuclides intended to image and study function of the GIT, certain assumptions must be made, most importantly that no radiolabel becomes detached from the tracer or leaves the GIT lumen. Also, that the tracer is neither secreted by parietal cells nor absorbable. ${ }^{16}$ Tc-99m labelled sulphur colloid fulfils these conditions, for extensive in vitro tests of this radiopharmaceutical's sta- bility in the presence of high molarity hydrochloric acid $(\mathrm{HCl})$ and acidified milk found insignificant amounts of free Tc-99m. ${ }^{17}$ These assumptions are therefore justified, so Tc-99m sulphur colloid is frequently used in these investigations, and is the typical radionuclide used in dosimetric calculations for GIT transit studies. The resulting absorbed doses depend on the residence times in various parts of the GIT. These values can be derived from a published model of GIT transit. $^{18}$ Transit times have been obtained for the paediatric ages under consideration (Stabin and Gelfand 1998). Absorbed doses have been obtained from paediatric phantoms using body morphology of 'average' children of the ages stated and the resulting phantom masses are 3.2, 5.5, 10.2, 18.5 and $32.4 \mathrm{~kg}$. Also, appropriate swallowed doses (Table I) for these five paediatric ages were required. Computations using these data result in absorbed radiation doses for the listed organs given in Table I. The quoted swallowed doses are 'average'and not necessarily those of specific patients. Even for very-low-birth-weight infants in the first 2 months of life, absorbed radiation doses from radiological studies are small compared with those which form the basis of risk estimates. ${ }^{19}$ There can be much uncertainty in the dosimetric results due to well known natural variation between subjects, for example in height and gut transit times. In adults it has been shown that when activity is in the stomach, the resulting $S$ values depend critically on the height of the model representing the patient. With a height variation of $12.5 \%, \mathrm{~S}$ values can vary by up to $48 \%{ }^{20}$

It is clear that there is a wide range of absorbed doses in different organs, which result in different risk factors. Hence the concepts of effective dose (ED) and effective dose equivalent (EDE) have been derived to allow for this variation. ED and EDE consider the biological effectiveness of the radiation being absorbed and also a weighted risk (WR) for the different irradiated organs. The WR for a given organ or tissue is derived from estimated risk of deleterious effects (in essence resulting neoplasms or genetic defects) of ionising radiation. In this way overall risks can be estimated if the individual organ doses and their
Table I. Radiation dose estimates (after Stabin and Gelfand10) in selected target organs for typically administered activities of swallowed Tc-99m sulphur colloid in different paediatric age groups using calculation procedures described in the text

\begin{tabular}{lccccc}
\hline Age (yrs) & $\mathbf{0}$ & $\mathbf{1}$ & $\mathbf{5}$ & $\mathbf{1 0}$ & $\mathbf{1 5}$ \\
\hline Swallowed activity (MBq) & 9.3 & 9.3 & 18.5 & 18.5 & 18.5 \\
Organ & & & & & \\
$\quad$ Distal colon wall (mGy) & 13 & 5.9 & 5.7 & 3.1 & 1.9 \\
$\quad$ Proximal colon wall (mGy) & 6.8 & 3.5 & 6.8 & 4.4 & 2.8 \\
Small gut (mGy) & 2.0 & 1.2 & 3.0 & 2.2 & 1.4 \\
Stomach wall (mGy) & 2.7 & 0.93 & 1.9 & 1.1 & 0.83 \\
Ovaries (mGy) & 1.1 & 0.68 & 1.2 & 0.83 & 0.57 \\
Testes (mGy) & 0.17 & 0.09 & 0,12 & 0.068 & 0.035 \\
Effective dose (mSv) & 2.1 & 1.1 & 1.5 & 0.91 & 0.58 \\
\end{tabular}


Table 10.5 Effective Dose Equivalent (EDE) for common CT examinations and the range of doses found in the 1989 survey by $\mathrm{NRPB}^{21}$. The corresponding contributions to the collective population dose are also tabulated

\begin{tabular}{lcccc}
\hline Examination & Mean EDE and range $(\mathrm{mSv})$ & Frequency of exams, \% & \% collective EDE \\
\hline Routine head & 3.49 & $(0.91-9.77)$ & 34.9 & 23 \\
Posterior fossa & 1.22 & $(0.33-3.68)$ & 8.1 & 1.9 \\
Pituitary & 1.1 & $(0.2-3.71)$ & 2.1 & 0.4 \\
IAM & 0.43 & $(0.06-2.09)$ & 2.2 & 0.2 \\
Orbits & 1.13 & $(0.2-5.62)$ & 1.9 & 0.4 \\
Facial bones & 0.69 & $(0.02-3.47)$ & 1 & 0.1 \\
Cervical spine & 1.94 & $(0.21-5.52)$ & 1.8 & 0.7 \\
Thoracic spine & 7.76 & $(0.85-24.3)$ & 0.7 & 1 \\
Routine chest & 9.13 & $(1.03-22)$ & 7.9 & 13.7 \\
Mediastinum & 7.39 & $(0.58-22.9)$ & 4 & 5.5 \\
Routine abdomen & 8.82 & $(1.86-25.2)$ & 11.6 & 19.3 \\
Liver & 10.2 & $(2.44-28.4)$ & 3.5 & 6.8 \\
Pancreas & 6.91 & $(0.92-22.4)$ & 2.7 & 3.4 \\
Kidneys & 8.62 & $(2.86-41.2)$ & 1.7 & 2.7 \\
Adrenals & 3.74 & $(0.58-14.7)$ & 1 & 0.7 \\
Lumbar spine & 5.98 & $(1.03-20.3)$ & 7 & 7.9 \\
Routine pelvis & 9.38 & $(1.49-32.1)$ & 5.6 & 9.9 \\
Others & & & 2.3 & 2.4 \\
\hline
\end{tabular}

Mean EDE (all examinations) $5.3 \mathrm{mSv}$; no. of examinations, 850 000; collective EDE, 4500 man-Sv

to give an overall range of doses between different hospitals for the same type of examination of $10-40$ times.

These results are summarized in Table 10.5, which also gives the collective Effective Dose Equivalent for the range of CT examinations, which was obtained from data on the frequency of the examinations found from the NRPB survey.

Typical doses from CT in the UK are large when compared with plain film radiography, and it can be seen that CT contributes the major component of the population dose from diagnostic X-rays, despite its relatively infrequent use, being approximately $20 \%$ of the 20000 man-Sv total collective dose.

It is therefore important that in view of the high dose levels, all CT referrals should be vetted by experienced radiologists as recommended by the Royal College of Radiologists and NRPB ${ }^{14,35}$. The large variation in doses between hospitals also points to the need for audit of the parameters selected for standard protocols, and for regular quality control and dosimetry checks on the performance of CT scanners.

\section{TYPICAL DOSES AND ESTIMATED RISKS FROM DIAGNOSTIC RADIOLOGY AND NUCLEAR MEDICINE EXAMINATIONS}

A summary of recently published data on Effective Doses in a variety of typical radiological and nuclear medicine examinations is given in Table 10.6. Estimated risks of induced fatal cancer or genetic effects, using the risk factors published by ICRP, are also given. It may be seen that the examinations which give rise to the greatest risk are those involving multiple radiographs, fluoroscopy of the abdomen, or CT examinations of the chest or abdomen, since these examinations give the greatest exposure and involve irradiation of the most sensitive organs.

In comparing average patient doses it is often valuable to put these in terms of more familiar units. Two methods employed are to quote the Effective Dose as a multiple of that received from a standard chest X-ray, or in terms of the length of time in which a similar dose would be received from natural background

Table 10.6 Typical Effective Doses to patients and calculated associated risks of fatal malignancy for various radiological examinations

\begin{tabular}{|c|c|c|c|c|c|c|c|c|}
\hline \multirow{3}{*}{ Examination } & \multicolumn{6}{|c|}{ Probability of radiation effect occurring $\left(\times 10^{-6}\right.$ [per million persons]) } & \multirow{3}{*}{$\begin{array}{l}\text { Effects on fetus } \\
\text { Fatal cancers }\end{array}$} & \multirow[b]{3}{*}{ Hereditary disease } \\
\hline & \multirow{2}{*}{$\begin{array}{l}\text { Average Effective } \\
\text { Dose Equivalent, } \\
m S v\end{array}$} & \multirow{2}{*}{$\begin{array}{l}\text { Average } \\
\text { fetal dose, } \\
m S v\end{array}$} & \multicolumn{2}{|c|}{ Fatal somatic } & \multicolumn{2}{|c|}{ Fatal genetic } & & \\
\hline & & & $M$ & F & $M$ & $F$ & & \\
\hline Chest & 0.05 & $<0.01$ & 0.27 & 0.47 & - & - & $<0.3$ & $<0.24$ \\
\hline Skull & 0.15 & $<0.01$ & 1.7 & 1.7 & - & - & $<0.3$ & $<0.24$ \\
\hline Thoracic spine & 0.92 & $<0.01$ & 7 & 11 & - & - & $<0.3$ & $<0.24$ \\
\hline Lumbar spine & 2.1 & 3.2 & 25 & 26 & 0.2 & 16 & 95 . & 77 \\
\hline Abdomen & 1.4 & 2.6 & 9.4 & 9.5 & 2 & 11 & 77 & 62 \\
\hline Pelvis & 1.2 & 1.7 & 3.9 & 3.9 & 24 & 6.3 & 51 & 40 \\
\hline IVU & 4.4 & 3.2 & 26 & 37 & 23 & 19 & 96 & 77 \\
\hline Barium meal & 3.8 & 2.8 & 26 & 31 & 0.8 & 9.4 & 84 & 67 \\
\hline Barium enema & 7.7 & 16 & 37 & 388 & 5.4 & 26 & 480 & 390 \\
\hline CT skull (12 slices) & 3.5 & $<0.005$ & 42 & 47 & - & - & $<0.15$ & $<0.12$ \\
\hline CT chest ( 24 slices) & 9.1 & 0.06 & 470 & 630 & - & - & 1.8 & 1.5 \\
\hline CT abdomen ( 30 slices) & 8.8 & 8 & 340 & 380 & - & - & 240 & 190 \\
\hline
\end{tabular}


WR values are known. ED and EDE values are usually similar, but their derivations differ according to the sizes of WR values used. They depend on organ doses that can be calculated using mathematical models of the body and simulated photon interactions. Their greatest use is for relative measures rather than absolute values. ${ }^{21}$

It is clear from Table I that the highest $D$ values are found in the large gut and that these values fall rapidly with age. This is due to the increasing length of the GIT with age, hence both transit times and distances to these organs from sources of radiation increase, although it must be reemphasised that transit times have a large natural variation. Ovaries receive greater irradiation than testes, due to their location, but even this is small.

\section{GIT radiology}

Radiology most frequently gives morphological information and is unsurpassed in this respect, so when anatomical abnormalities are sought, it has no equal. Barium (Ba) studies allow for observation of GIT motility, but otherwise their functional assessment is limited.

The principle of radiology is well known. A collimated beam of X-rays is directed from an external source onto the region to be investigated and the radiation, which traverses the patient, is recorded. Thus a map of local absorption variation is obtained and such images are the study's end products. In general, radiological images are obtained from film exposure or by digital means. Usually the latter provides lower $\mathrm{D}$ values. In digital radiography final images are affected by many more factors than in film radiography, so it can be difficult to estimate correct exposure. ${ }^{22}$ Despite more than a century of radiology, precise determination of image quality is still under discussion. Physical measures are the best contenders to describe such quality. ${ }^{23}$ It is therefore essential to optimise examination practice and equipment. ${ }^{24}$

Radiological absorbed doses depend on many factors (physical and other), with very complex interrelationships. ${ }^{25}$ A considerable trade-off between dose restriction and image quality is usually necessary. In gener$\mathrm{al}$, there is a lack of data on the relation between quality of image and D, especially with regard to paediatrics, in both radiology and nuclear medicine. A programme of the European Union (EU) to address this has been set up, with emphasis on paediatric fluoroscopy. ${ }^{26}$ Although digital fluoroscopy has many advantages, high exposure can result from larger numbers of images recorded, consequently the FDA recommends that the fluoroscopy dose be continuously displayed. ${ }^{23}$ Ionising radiation absorbed by the patient depends on the total irradiation time. Fluoroscopy has the potential to result in high absorbed radiation doses.

In GIT studies, a Ba compound is normally swallowed for radiological investigations. (It should be noted that this is an unphysiological material with high density. Only those suffering from pica swallow comparable substances. In contrast, the scintigraphic investigation is totally physiological, with a small fraction of a gram of tracer swallowed mixed with normal food or drink.) The radiological study can image all stages of deglutition of the contrast medium, its residence in the stomach, possible GOR and gastric emptying, by recording images of the contrast medium at different times. Quantitation of the amount of contrast medium is not possible, for it is effectively either present or not in images, and so a wide range of amounts of contrast medium cannot be recognised.

\section{Radiological dosimetry}

There are significant differences between irradiation in radiology and scintigraphy. In the former the source of the radiation is an X-ray tube outside the patient. The radiation is directed in straight lines within a cone before interacting with tissue, and the region irradiated is usually well circumscribed. Hence compared with nuclear medicine, biological activity in organs and tissues does not play a role in obtaining an absorbed dose after a radiological study. It is the relative positions, nature and absorptive characteristics of organs and intervening tissues that are important instead. For these reasons some different physical quantities are' needed compared with scintigraphic dosimetry. These include entrance skin dose (ESD), the radiation absorbed by the skin at the beams's entry into the patient and kerma (the Kinetic Energy Released per unit Mass in Air, or other materials, and measured in Gy). Estimation of organ doses, or effective doses in radiology, is usually accomplished by appropriate measurement.

Intracavitary detectors in all organs of interest are clearly impracti$\mathrm{cal}$, as is mapping a total skin dose. Often a dose-area product (DAP) $\left(\mathrm{Gy} / \mathrm{cm}^{2}\right)$, is measured using an ionisation chamber, when all irradiation parameters are not known. ${ }^{27}$ The DAP is the product of an entrance dose at a reference distance and the field size. It 


\section{ORIGINAL ARTICLE}

is independent of the X-ray source to patient distance. Individual organ doses can vary with many beam-associated factors (including projection and collimation), but the image detection process and patient characteristics do play a role. A combination of measured entry exposure (energy transfer) and appropriate conversion factors $^{28}$ allows estimation of absorbed doses.

For many years radiological doses were expressed in terms of measured doses (ESD, etc.). ${ }^{29}$ However, in 1996 international diagnostic reference levels were published ${ }^{30}$ and amplified a year later by EU directives. ${ }^{31}$ These use readily available dosimeters, depending on ESD and DAP. Yet often these guideline levels are consciously exceeded after clinically justified judgements. In South Africa (E Hering - personal communication, 2001) and many other countries, they are not yet used.

Fluoroscopy involving image intensifiers is often used in GIT studies to obtain real-time images. Image contrast in fluoroscopy can be quantified $^{32}$ and this greatly aids the optimisation of image quality. Standard fluoroscopy has an entry exposure limit of $0.1 \mathrm{~Gy} / \mathrm{min}$, although many devices allow this to be exceeded. ${ }^{33}$ A pharyngeal videofluoroscopy unit which investigates swallowing dysfunction has been shown to have a mean ED of $0.4 \mathrm{mSv}$ based on a DAP measurement, ${ }^{34}$ yet this imaging study provides only a small amount of information compared with the total gastro-intestinal scintigraphy study. With regard to adults, an examination of over $10000 \mathrm{Ba}$ studies showed that a reduction of DAP of about $50 \%$ is found when studies using digital equipment are compared with those using non-digital equipment. ${ }^{35}$ In the USA the extent of radiographic dose variation for studies done under comparable fluoroscopic conditions is alarming. A study of 400 different units using the same test objects found that entrance air kerma rates varied from 10 to $130 \mathrm{mGy} / \mathrm{min}^{36}$

After measurements are made, calculations can provide $D$ values for different organs. ${ }^{37}$ Specific calculations have been derived for paediatric situations $s^{38,39}$ and influence of patient size was included later..$^{40}$ Thus conversion coefficients for use with measured air kerma, ESD or DAP allowed organ equivalent dose to be obtained. ${ }^{41}$ Published conversion factors obtained from two different programmes, $\mathrm{PCXMC}^{42}$ and a National Radiation Protection Board (NRPB) publication, ${ }^{43}$ both using Monte Carlo techniques, show very close agreement for patients aged $0,1,10$ and 15 years. They relate air kerma to ED for a D value in bone marrow, with values of 400 - $650 \mathrm{~Gy} / \mathrm{Sv}$ for the different ages. But in using these values individual patient variation (e.g. thickness) must often be considered. Apart from this, many physical conditions of the irradiation procedure (e.g. field size and position, photon spectrum, sourcefilm distance) can influence the resulting D values. ${ }^{37}$

National quality control programmes for paediatric radiology exist only in five European countries. However, it should be noted that in 1990 and 1991 vast variations in ESD for abdominal studies done on infants and 5-year-old patients were described, ${ }^{44}$ with minimum to maximum ratios of 1:42 and 1:52 for these two age groups respectively. The subsequent $\mathrm{EU}$ directives ${ }^{31}$ therefore represent an important advance in paedi- atric radiology. Reference values for paediatric fluoroscopy in a $\mathrm{Ba}$ meal are exposure time 2 minutes and total DAP $0.4 \mathrm{~Gy} / \mathrm{cm}^{2}$. However, it should be recalled that paediatric patients are very variable, with thickness varying up to twofold in any age band. ${ }^{24}$

Quantitative considerations with regard to radiation dosimetry in fluoroscopy involving paediatric patients are beset with difficulties. These include relatively few data available, wide ranges in patient size and a variety of different age ranges in use, or none. ${ }^{45}$ Current consenus is to take measurements with thermoluminescent detectors (TLDs) and use DAP more often in paediatric fluoroscopy. Recent work with lithium fluoride (LiF) TLD dosimeters has shown them to be particularly suited for paediatric dosimetry, with good resolution, lower detection limits than previous types of TLD and excellent linearity. ${ }^{46}$

In applying experimental data from phantom measurements to paediatric radiology, ${ }^{45}$ a size correction was made first. ${ }^{47}$ In all, 804 measurements were taken in patients aged from 0 to 15 years. This resulted in reference values of DAP for five age ranges, given in Table II.

Twelve randomly selected Ba swallows performed at this institution, in patients with ages ranging from 7 months to 13 years, were studied to allow determinations of their total exposure times and areas irradiated. The times ranged from 1.8 to $7.0 \mathrm{~min}$ utes and the areas ranged between 264 and $4896 \mathrm{~cm}^{2}$. Hence dose levels of resulting irradiations could be calculated from measured radiation levels for the equipment (E Smit - personal communication, 2001), assuming that typical settings of the equipment 


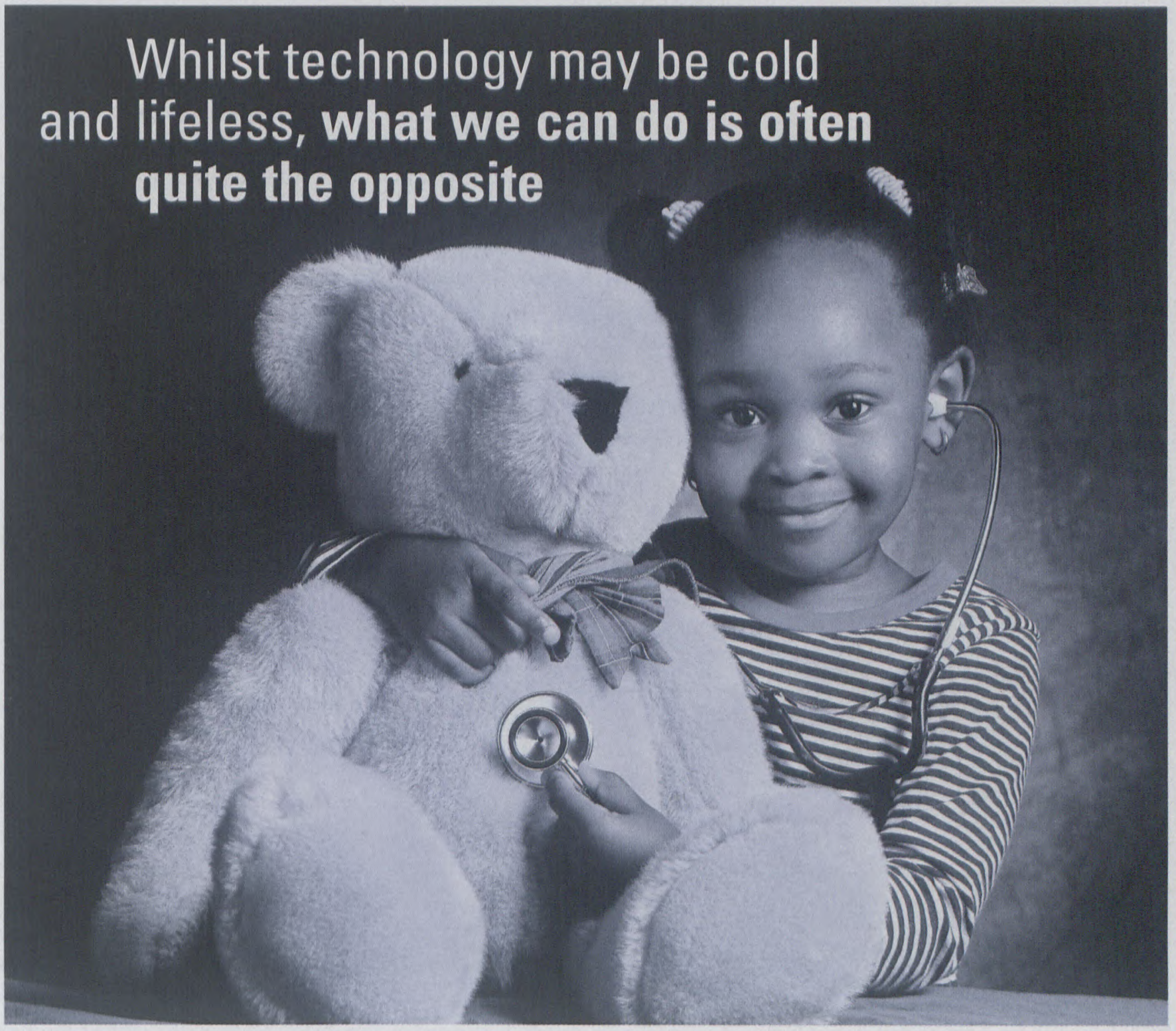

We always incorporate the care of both patient and doctor in the design of all Siemens medical equipment. This allows you to make more accurate and efficient diagnoses that result in a more rewarding relationship with your patients.

An excellent example of Siemens partnership with you can be seen in the unique CARE features of our equipment.
CARE is based on providing the least invasive diagnosis and treatment procedures for patients and clinicians, while maintaining the same excellent image quality.

Siemens believes in a lifelong partnership with all our medical clients which goes way beyond the mere supply of advanced technical medical equipment. 


\section{ORIGINAL ARTICLE}

(which were recorded for all the studies) were providing $50 \%$ of the maximum possible dose rate. These doses are given in Table III.

\section{Discussion}

The two study modalities under consideration provide different and complementary information. At recent large nuclear medical congresses 'few papers .... dealt with dosimetry $\ldots{ }^{48}$ Yet dosimetry, especially in paediatrics, is of critical importance, although relatively little has been published on this subject, in part presumably because of its complexity and the

general belief that (in nuclear medicine at least) the potential damage is far outweighed by advantages of the study. However, there is much more reported work on calculation and measurement of dosimetry in scintigraphy than radiology. Also the scintigraphic mathematical models and their application are more detailed. This is probably because of the general public's concern whenever radioactivity is encountered and the fear of harmful consequences. Nuclear medicine practitioners usually deliberate more on dosimetric considerations than radiologists. This is probably

Table II. Reference dose levels for paediatric Ba meals and swallows (adapted from Chapple et al., 1998) ${ }^{45}$

\begin{tabular}{lcc}
\hline & \multicolumn{2}{c}{ Dose area products $\left(\mathrm{Gy} / \mathrm{cm}^{2}\right)$} \\
\cline { 2 - 3 } Age range $(\mathrm{yrs})$ & Barium meal & Barium swallow \\
\hline Neonate & 1.082 & 1.191 \\
Infants & 1.561 & 1.714 \\
$1-5$ & 2.074 & 2.449 \\
$6-10$ & 3.290 & - \\
$11-15$ & 5.820 & -
\end{tabular}

Table III. Details of Ba swallow studies in patients from the Red Cross War Memorial Children's Hospital

\begin{tabular}{lccc}
\hline Age & $\begin{array}{c}\text { Total area } \\
\text { irradiated }(\mathbf{c m} 2)\end{array}$ & $\begin{array}{c}\text { Duration of } \\
\text { irradiation }(\mathbf{m i n})\end{array}$ & $\begin{array}{c}\text { Irradiation DAP } \\
\left(\mathbf{m G y} / \mathbf{c m}^{2}\right)\end{array}$ \\
\hline 7 mo & 264 & 1.8 & 187.3 \\
$8 \mathrm{mo}$ & 873 & 1.7 & 585.1 \\
$8 \mathrm{mo}$ & 1082 & 5.6 & 2389 \\
4y & 576 & 2.4 & 545.0 \\
5 y & 3630 & 3.7 & 5295 \\
5 y 6 mo & 851 & 1.7 & 570.3 \\
5 y 10 mo & 1136 & 6.7 & 3001 \\
8 y & 1159 & 2.0 & 913.8 \\
8 y 10 mo & 1973 & 1.8 & 1400 \\
9 y 8 mo & 2520 & 3.4 & 3378 \\
11 y 10 mo & 1854 & 3.4 & 2485 \\
13y & 4896 & 7.0 & 13511 \\
\hline
\end{tabular}

because radiology has a far longer history and in the public view is considered innocuous by comparison. In the training of radiologists there is relatively little emphasis given to dosimetry in contrast to nuclear medicine.

Although typical organ doses have been calculated for radiological studies, these tend to be for adults ${ }^{27}$ and little comparable work has been done on children. However, it is clear that the resulting doses for abdominal Xrays are about an order of magnitude greater than for scintigraphy. ${ }^{10,27}$

It is clear that the DAP values for the $\mathrm{Ba}$ swallows in our institution (Table III) are mostly, but not all, below those given as recommended values (Table II). It is anticipated that this will improve significantly when new digital equipment is installed in the near future. It is equally clear that there is a great variation in the total irradiation times (from 1.7 to 7.0 minutes) and also in the areas irradiated (which depend on the patient's size and the number of images recorded). Confirmation of the general observation that comparable studies using scintigraphy and radiology have much smaller $\mathrm{D}$ values for the former are certainly confirmed here for paediatric values when Tables I and III are compared.

An ultrasound study is also available to detect reflux ${ }^{49}$ with a zero D value, but it has severe limitations compared with contrast radiography and scintigraphy. Although a patent gastro-oesophageal junction can be observed and its diameter recorded (which the other modalities cannot do), the reflux cannot be seen after rising the first centimetre or so, hence its height, directly related to its severity, remains unknown. 


\section{Conclusions}

Several clear conclusions result from this review. The two modalities, scintigraphy and radiology, provide different information and so complement each other with regard to foregut studies. The nature of the radiological examination is such that the resulting absorbed radiation dose can vary greatly. Even when reference doses are in common use, which will be in the near or midterm future, such reference dose values will be consciously exceeded when it is decided that clinical needs so dictate. In scintigraphy there is little variation in $\mathrm{D}$, whatever the clinical circumstances of any one individual patient, for a standard dose of radiotracer is administered. Quantitative understanding of the resulting $\mathrm{D}$ values is greater in nuclear medicine than radiology because of greater caution in using gamma rays than X-rays, but for historical rather than scientific reasons. However, the increasing use of digital radiology and rapidly advancing technology in image intensifiers makes it very likely that $\mathrm{D}$ values in radiology will steadily decrease in the next few decades. Gamma camera technology is also advancing, with sensitivity increasing, but currently at a slower rate than comparable radiological innovations. Finally, the resulting D values for scintigraphy are clearly less than those of analogous radiological studies and this is likely to remain true for some years to come.

\section{Acknowledgements}

We are grateful to Ms Ann Murray for help in selecting the radiographs used to produce Table III.

\section{References}

1. Roebuck. DJ. Risk and benefit in paediatric radiology. Pediatr Radiol 1999; 29: 637-640

2. Baulieu F, Maurage C, Baulieu JL, et al. A quantitative approach to gastro oesophageal reflux in children. Nucl Med Commun 1984; 5: 689-695

3. Davies RP, Morris LL, Savage JP, et al. Gastrooesophageal reflux: the role of imaging in diagnosis and management. Australas Radiol 1987; 31: 157-163.

4. Paton JY, Cosgriff PS, Nanayakkara CS. The analytical sensitivity of Tc-99m radionuclide "milk" scanning in the detection of gastrooesophageal reflux. Pediatr Radiol 1985; 15: 381-383.

5. Zanzonico PB. Internal radionuclide radiation dosimetry: a review of basic concepts and recent developments. J Nucl Med 2000; 41: 297 . 308.

6. Loevinger R, Budinger TF, Watson EE. MIRD Primer for Absorbed Dose Calculations. New York: Society of Nuclear Medicine, 1991.

7. Cristy M, Eckerman K. Specific absorbed fractions of energy at various ages from internal photon sources (I - VII). Oak Ridge National Laboratory Report, ORNL/ TM-8381/V1-7. Springfield, Va., National Technical Information Service, 1987

8. Simpkin DJ. Radiation interactions and internal dosimetry in nuclear medicine. Radiographics 1999; 19: 155-167.

9. Toohey RE, Stabin MG, Watson EE. The AAPM/RSNA physics tutorial for residents: internal radiation dosimetry: principles and applications. Radiographics 2000; 20: 533-546.

10. Stabin MG, Gelfand MJ. Dosimetry of pediatric nuclear medicine procedures. Q J Nucl Med 1998; 42: 93-112.

11. Thierens HM, Monsieurs MA, Brans B, et al Dosimetry from organ to cellular dimensions. Comput Med Imaging Graph 2001; 25: 187-193.

12. Piepsz A, Hahn K, Roca I, et al. A radiopharmaceutic schedule for imaging in paediatrics. Eur I Nucl Med 1990; 17: 127-129.

13. Treves ST. Introduction. In Treves ST, ed. Pediatric Nuclear Medicine, 2nd ed. New York: Springer-Verlag, 1995: 1-11.

14. Anon. Administration of Radioactive Substances Advisory Committee. Notes for Guidance on the Administration of Radioactive Substances to Persons for Purposes of Diagnosis, Treatment or Research. Chilton, Oxon, UK: National Radiation Protection Board, 1998.

15. Smith T, Gordon I. An update of radiopharmaceutical schedules in children. Nucl Med Commun 1998; 19: 1023-1036.

16. Fisher RS, Malmud S, Roberts GS, Lobis IF. Gastroesophageal (GE) scintiscanning to detect and quantitate GE reflux. Gastroenterology 1976; 70: 301-308.

17. Heyman S, Kirkpatrick JA, Winter HS, et al. An improved radionuclide method for the diagnosis of gastroesophageal reflux and aspiration in children (milk scan). Radiology 1979; 131; 479. 482

18. Anon. International Commission on Radiological Protection. Limits for Intakes of Radionuclides by Workers. IRCP Publication 30. New York: Pergamon Press, 1979.
19. Wilson-Costello D, Rao PS, Morrison S, et al Radiation exposure from diagnostic radiographs in extremely low birth weight infants. Pediatrics 1996; 97: 369-374.

20. Clairand I, Boucher LG, Ricard M, et al Improvements in internal dose calculations using mathematical models of different adult heights. Phys Med Biol 2000; 45: 2771-2785.

21. McCollough $\mathrm{CH}$, Schueler BA. Calculation of effective dose. Med Phys 2000; 27: 828-837.

22. Busch HP, Jaschke W. Adaptation of the quality criteria concept to digital radiology. Radiat Prot Dosim 1998; 80: 61-63.

23. Oestmann JW. The role and impact of reference doses in diagnostic radiology: problems and perspectives. Radiat Prot Dosim 1998; 80: 21-22.

24. Gfirtner H, Giesse E, Schmidt T. Dosimetric methods for and influence of exposure parameters on the establishment of reference doses for examination using fluoroscopy. Radiat Prot Dosim 1998; 80: 121-128.

25. Schueler BA. Clinical application of basic $\mathbf{x}$-ray physics principles. Radiographics 1998; 18: 731744.

26. Anon. International Commission on Radiological Protection. Basic principles in assessment and selection of reference doses. Radiat Prot Dosim 1998; 80: 331-335.

27. Rassow S. From the entrance dose to the calculation of organ doses. Radiat Prot Dosim 1998; 80: 327-329.

28. Rosenstein M. Handbook of Selected Tissue Doses for Projections Common in Diagnostic Radiology. Rockville, Md.: US Food and Drug Administration, Center for Devices and Radiological Health, 1988

29. Wall BF, Shrimpton PC. The historical development of reference doses in diagnostic radiology. Radiat Prot Dosim 1998; 80: 5-20.

30. Anon. International Commission on Radiological Protection. Radiological Protection and Safety in Medicine. ICRP publication 73 Oxford, UK: Elsevier, 1996.

31. Anon. European Commission. Council Directive 97/43/Euratom of 30 June 1997 on 'Health protection of individuals against the dangers of ionising radiation in relation to medical exposure' and Repealing Directive 84/466/Euratom. Official Journal of the European Communities. No. L 180/22 (1997).

32. Taubel JP, Schueler BA, Vrieze TJ, et al. Quantification of fluoroscopic imaging system contrast by using video wave form monitoring Radiographics 2001; 21: 501-508.

33. Parry RA, Glaze SA, Archer BR. Typical patient radiation doses in diagnostic radiology. Radiographics 1999; 19:1289-1302

34. Wright RE, Boyd CS, Workman A. Radiation doses during pharyngeal videofluoroscopy. Dysphagia 1998; 13: 112-115.

35. Broadhead DA, Chapple CL, Faulkner K. The impact of digital imaging on patient doses during barium studies. Br J Radiol 1995; 68: 992 996.

36. Verdun FR, Capasso P, Valley JF, et al. Dose evaluation in fluoroscopy. Radiat Prot Dosim 1998; 80: 139-141.

37. Zankl M. Methods for assessing organ doses using computational models. Radiat Prot Dosim 1998; 80: 207-212 


\section{ORIGINAL ARTICLE}

38. Chen WL, Poston JW, Warner GG. An Evaluation of Absorbed Dose in Child Phantoms Exposed to Diagnostic Medical X-rays. Report no. ORNL/TM-5933. Oak Ridge, Tenn.: Oak Ridge National Laboratory, 1978.

39. Rosenstein M, Beck TJ, Warner GG. Handbook of Selected Organ Doses From Projections Common in Pediatric Radiology. Rockville, Md.: HEW publication (FDA) No. 79-8079, Rockville Md.: 1979.

40. Veit R, Zankl M. Variation of organ doses in pediatric radiology due to patient diameter, calculated with phantoms of varying voxel size. Radiat Prot Dosim 1993; 49: 353-356.

41. Hart D, Jones DG, Wall BF. Coefficients for Estimating Effective Doses From Paediatric X-ray Examinations. Report no. NRPB- R279. Chilton, Oxon, UK.: National Radiation Protection Board, 1996a.
42. Servomaa A, Tapiovaara M. Organ dose calculation in medical $x$ ray examinations by the program PCXMC. Radiat Prot Dosim 1998; 80: 213-219.

43. Hart D, Jones DG, Wall BE. Normalised Organ Doses for Paediatric X-ray Examinations Caluculated Using Monte Carlo Techniques. Report no. NRPB- SR279. Chilton, Oxon, UK.: National Radiation Protection Board, 1996b.

44. Perlmutter N, Arthur RJ, Beluffi G, et al. The quality criteria for diagnostic radiographic images in paediatrics, Radiat Prot Dosim 1998; 80: 45-48.

45. Chapple CL, Broadhead DA, Faulkner K. Reference doses for paediatrics from fluoroscopic procedures. Radiat Prot Dosim 1998; 80: 203-206.

46. Fill UA, Regulia DF. Advanced LiF technology for the assessment of patient exposure in diagnostic radiology. Radiat Prot Dosim 1998; 80. 225-229.

47. Chapple CL, Broadhead DA, Faulkner K Phantom based method for deriving typical patient doses from measurements of dose-area product on populations of patients. Br J Radio 1995; 68: 1083-1086.

48. Jacobsson H, Gryback P, Larsson SA. What is the message of a meeting? Analysis and comparison of the 2458 abstracts of the EANM/ WCNMB congress in Berlin and the SNM meeting in Toronto in 1998. Eur J Nucl Med $2000 ; 27: 215-222$

49. Wynchank S, Mann MD, Fisher R, et al Ultrasound as a screening study for gastro oesophageal reflux in children. Ann Trop Paediatr/Int Child Health 1997; 17: 343-348.

\section{The Practice of Mammography}

Pathology, Technique, Interpretation, Adjunct Modalities Dronkers et al.

Even with the advent of many new radiographic techniques, mammography remains the most important and effective method of breast examination available today. This outstanding guide, using anatomy and pathology as a starting point, gives clear and accurate instructions on all facets of performing breast mammograms, from proper positioning to analysing the findings. In addition, this guide focuses on the non-radiological aspects of breast carcinoma such as clinical diagnosis, treatment, epidemiology, and risk factors. You'll also learn about screening methods to detect and avoid potential problems. The instructive nature of this text makes it an excellent -resource for students and residents. Radiologists and OB/GYNs who need a quick update or refresher will also appreciate its value. Here is the book that belongs on the shelves of every medical school library, residency program, and specialist's office!

Sept 2001, 266 pp, 492 illus., hardcover, R1 199

\section{The Electronic Clinical Brain Atlas \\ Multi-Planar Navigation of the Human Brain \\ Nowinski / Nowinski / Raghavan}

This CD-ROM integrates several landmark print atlases as well as MR scans into a multi-purpose, multi-dimensional, interactive clinical tool. The work incorporates the following classic Thieme atlases: Co-Planar Stereotaxic Atlas of the Human Brain by Talairach \& Tournoux; Referentially Oriented Cerebral MRI Anatomy by Talairach \& Tournoux; and Atlas for Stereotaxy of the Human Brain by Schaltenbrand and Wahren. Users will find the original information contained in the print atlases, all digitised, preprocessed, enhanced and fully labeled. With its unique scaling of images, this CD-ROM greatly facilitates the correlation of specific anatomical structures from the atlas to actual patient scans.

1998, manual 50 pp, CD-ROM, R3 999

\section{Cerebral MR Perfusion Imaging Principles and Current Applications \\ Sorensen / Reimer}

This book will familiarise the reader with the basic principles of perfusion MR imaging. Relevant technical aspects, contrast agents and the postprocessing of images are presented, and imaging protocols are provided. Dedicated software for personal use on the postprocessing of images is provided on a CD-ROM containing hundreds of images and movie clips that demonstrate all concepts. In addition to the technical details of acquisition and postprocessing, numerous examples of the application of these tools in the clinical setting are also shown. In particular, the book includes a discussion of the role of perfusion MRI in the current evaluation of cerebrovascular disease, including an integrative approach using diffusion in conjunction with perfusion imaging. Covers:

$\checkmark$ all relevant technical aspects of perfusion MR imaging

$\checkmark$ imaging protocols

$\checkmark$ application of these tools in a clinical setting

$\checkmark$ integration of diffusion imaging with perfusion imaging for enhanced diagnostic capabilities

It also addresses the role of perfusion MRI in the assessment of cerebral neoplasia, challenges and opportunities for treatment that tumors present, and the particular strengths of perfusion MRI, such as its relatively high resolution and possible microvascular specificity. For newcomers and experienced practitioners, this is a "nuts-and-bolts" description of an important new technique.

System Requirements: MS Windows (all versions), IBMcompatible PC with 486 processor or higher and 8 MB RAM, Super VGA graphics adapter (min. 256 colors), CD ROM player. 2001, 160 pp, 235 illus., hardcover book + CD-ROM, R950

Orders: The South African Medical Association

Private Bag X1, Pinelands 7430

Tel (021) 530-6527, fax (021) 531-4126

E-mail: fpaim@samedical.org

Allow 3-4 weeks for delivery. 